\title{
BMJ Open Recombinant human TNK tissue-type plasminogen activator (rhTNK-tPA) versus alteplase (rt-PA) as fibrinolytic therapy for acute ST-segment elevation myocardial infarction (China TNK STEMI): protocol for a randomised, controlled, non-inferiority trial
}

\author{
Hai-bo Wang, ${ }^{1}$ Ping Ji, ${ }^{2}$ Xing-Shan Zhao, ${ }^{3}$ Haiyan Xu, ${ }^{4}$ Xiao-Yan Yan, ${ }^{1}$ Qin Yang, \\ Chen Yao, ${ }^{1}$ Run-Lin Gao, ${ }^{4}$ Yang-feng Wu, ${ }^{1}$ Shu-Bin Qiao ${ }^{4}$
}

To cite: Wang H, Ji P, Zhao X-S, et al. Recombinant human TNK tissue-type plasminogen activator (rhTNK-tPA) versus alteplase (rt-PA) as fibrinolytic therapy for acute ST-segment elevation myocardial infarction (China TNK STEMI): protocol for a randomised, controlled, non-inferiority trial. BMJ Open 2017;7:e016838. doi:10.1136/ bmjopen-2017-016838

- Prepublication history for this paper is available online. To view these files please visit the journal online (http://dx.doi org/10.1136/bmjopen-2017016838).

$\mathrm{H}-\mathrm{W}$ and $\mathrm{X}-\mathrm{SZ}$ contributed equally.

Received 15 March 2017 Revised 6 July 2017 Accepted 7 July 2017

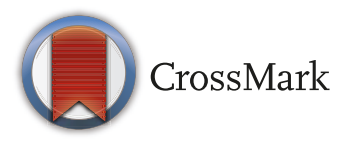

For numbered affiliations see end of article.

Correspondence to

Dr Yang-feng Wu;

wuyf@bjmu.edu.cn and Dr Shu-

Bin Qiao;

qsbfw@sina.com

\section{ABSTRACT}

Aim To evaluate the efficacy and safety of recombinant human TNK tissue-type plasminogen activator (rhTNKtPA) in lowering major adverse cardiovascular and cerebrovascular events (MACCEs) in Chinese acute STsegment elevation myocardial infarction (STEMI) patients. Methods and analysis The study is designed as a multicentre, randomised, controlled non-inferiority phase IV trial with balanced randomisation $(1: 1)$ in patients with STEMI. The planned sample size is 6200 participants (or 3100 per arm). Participants with STEMI will be randomised to receive either rhTNK-tPA or alteplase (rt-PA), with stratification by research centre, age and the time from symptom onset to randomisation. All patients will receive concomitant antiplatelet and anticoagulant therapy before fibrinolytic therapy. The participants assigned to the intervention group will receive an intravenous bolus of $16 \mathrm{mg}$ rhTNK-tPA, while those assigned to the control group will receive an intravenous bolus of $8 \mathrm{mg}$ rt-PA followed by $42 \mathrm{mg}$ infusion over 90 mins. Other medications can also be administered at the discretion of the cardiologists in charge. All participants will be followed up for the primary study endpoint, the occurrence of MACCEs within 30 days after fibrinolytic therapy, which is defined as all-cause mortality, non-fatal re-infarction, nonfatal stroke, percutaneous coronary intervention (PCI) due to thrombolysis failure, and $\mathrm{PCI}$ due to reocclusion. Both intention-to-treat and per-protocol analyses will be done for the primary analyses.

Ethics and dissemination The study procedures and informed consent form were approved by all participating hospitals. The results will be disseminated in peer review journals and academic conferences. This multicentre randomised controlled trial will provide high-quality data about the efficacy and safety of rhTNK-tPA and, once approved, its easier use should help improve the application of reperfusion therapy and hence the treatment outcomes of STEMI patients.

Trial registration number NCT02835534.
Strengths and limitations of this study

- Recombinant human TNK tissue-type plasminogen activator (rhTNK-tPA) has been newly developed by a Chinese company.

- This is the first randomised controlled trial evaluating the efficacy and safety of rhTNK-tPA among STsegment elevation myocardial infarction (STEMI) patients in China.

- Over 6200 participants will be recruited in about 150 hospitals in China.

- Pharmacoeconomic evaluation and economic analysis will be undertaken during the study.

\section{BACKGROUND}

The total number of cardiovascular and cerebrovascular deaths is increasing due to the ageing population and changing lifestyles, accounting for about $30 \%$ of all deaths globally in $2013^{1}$ and over $40 \%$ in China. ${ }^{2}$ ST-segment elevation myocardial infarction (STEMI), defined as full thickness damage of cardiac muscle resulting from occlusion of the coronary arteries by blood clot, is one of the most serious cardiovascular diseases with high mortality, ${ }^{3}$ and has become a major public health problem in China. ${ }^{4}$

Obtaining timely reperfusion of the infarct-related artery (IRA) by restoring coronary flow as early as possible is the critical element to reduce mortality and morbidity of STEMI patients. ${ }^{356}$ For STEMI patients, percutaneous coronary intervention (PCI) improves patient survival earlier and is associated with better outcomes compared with thrombolytic drugs. ${ }^{7-10}$ However, a large 
proportion of patients are unable to undergo PCI due to various reasons, especially in settings where PCI is not accessible within the required time frame, or is not affordable. ${ }^{11}{ }^{12}$ In addition, there is evidence that within 3 hours of onset fibrinolytic therapy is as effective as PCI therapy. ${ }^{13}$ Therefore, fibrinolytic therapy continues to be a preferable choice for those STEMI patients with poor access to healthcare.

Rapid infusion of the recombinant human tissue-type plasminogen activator (rt-PA) alteplase, combined with aspirin and heparin, is the most stable and credible strategy for fibrinolytic therapy among STEMI patients, showing significant effects in reperfusion of the IRA, protecting left ventricular function and reducing mortality. ${ }^{14}$ However, rt-PA has relatively quick plasma clearance and a short half-life; therefore, a high dose intravenous infusion is required to maintain effective drug concentration, which can prove inconvenient in the first-aid situation. Recombinant human TNK tissuetype plasminogen activator (rhTNK-tPA) is a genetically engineered variant of $\mathrm{rt}-\mathrm{PA}$ with better fibrin specificity. ${ }^{15}$ Tenecteplase is also a recombinant TNK-tPA that the US Food and Drug Administration (FDA) approved in 2000; it has significant advantages compared with rt-PA, including ease of administration, longer half-life and higher fibrin specificity. ${ }^{14}$ Assessment of the Safety and Efficacy of a New Thrombolytic Regimen (ASSENT-3) trial showed that the mortality rates at 1 year after acute myocardial infarction (MI) were $7.9 \%$ in the full-dose tenecteplase plus heparin group, $8.1 \%$ in the full-dose tenecteplase plus enoxaparin group, and $9.3 \%$ in the half-dose tenecteplase plus abciximab group. ${ }^{16}$

So far, rt-PA has been approved by China FDA, but tenecteplase has not yet entered the Chinese market. rhTNK-tPA (Recomlyse), which has the same amino acid sequence as tenecteplase, has been developed by Guangzhou Recomgen Biotech Co, Ltd, China. A previous phase II trial of rhTNK-tPA found that in comparison with rt-PA, ${ }^{17}$ Thrombolysis In Myocardial Infarction (TIMI) flow grade 2-3 in the IRA was significantly increased in the rhTNK-tPA group compared with the rt-PA group $(82.8 \%$ vs $67.4 \%)$. To further understand the efficacy and safety of rhTNK-tPA in reducing clinical events, a study with a large sample is to be conducted among STEMI patients in approximately 150 hospitals across China.

\section{STUDY DESIGN}

\section{Trial design and setting}

The study is designed as a multicentre, randomised, open-label, controlled non-inferiority phase IV trial with balanced randomisation (1:1) in patients with STEMI. The protocol of the current study was reported as adhering to the 'Standard Protocol Items: Recommendations for Interventional Trials' statement. After providing written informed consent, all eligible participants will be randomly assigned to receive fibrinolytic therapy either with rhTNK-tPA or rt-PA. Central randomisation is carried out by an interactive web-based system using the dynamic allocation method stratified by the research centre, age ( $\leq 60$ years vs $>60$ years), and the duration from onset of symptoms to randomisation ( $\leq 3$ hours vs $3-6$ hours). Participants are recruited continuously by physicians who are responsible for fibrinolytic therapy in approximately 150 hospitals across the country.

\section{Ethical approval}

The study procedures and informed consent form were approved by all participating hospitals, and the study was registered at www.clinicaltrials.gov (NCT02835534) on 12 June 2016 and updated on 13 July 2016. However, the registry indicated that the trial was retrospectively registered as the first participant was enrolled on 11 July 2016. Figure 1 demonstrates the study process for both the intervention and control groups.

\section{Study population}

A total of 6200 participants will be recruited from May 2016 in about 150 hospitals in China. To be eligible, participants should meet all of the following criteria: (1) aged 18-70 years; (2) diagnosed as acute STEMI, presenting with typical ischaemic chest pain lasting for $\geq 30 \mathrm{mins}$ and $\geq 0.1 \mathrm{mV}$ ST-segment elevation in $\geq 2 \mathrm{limb}$ leads or $\geq 0.2 \mathrm{mV}$ ST-segment elevation in $\geq 2$ contiguous precordial leads; (3) duration from onset of symptoms (typical ischaemic chest pain) to randomisation $\leq 6$ hours; (4) unable to undergo primary PCI within 1 hour or expected door-to-balloon time $>90$ mins; (5) willing to participate and provide written informed consent.

Patients will be excluded if any of the following criteria are met: (1) diagnosed with any of the following conditions: non-ST-segment-elevation acute MI or unstable angina pectoris; reinfarction; cardiogenic shock; suspected aortic dissection; new-onset left bundle branch block diagnosed by ECG; (2) having any contradiction to fibrinolysis (according to the Chinese Guideline for Diagnosis and Treatment of STEMI, 2015 edition), including: systolic blood pressure $>180 \mathrm{~mm}$ $\mathrm{Hg}$ and/or diastolic blood pressure $>110 \mathrm{~mm} \mathrm{Hg}$, and unresponsive to blood pressure-lowering treatment; history of intracerebral haemorrhage, cryptogenic stroke, or cerebral ischaemic stroke within 3 months; abnormality in cerebral vascular structure or intracranial malignant tumours; active haemorrhage, high risk of haemorrhage, or active peptic ulcer disease; severe facial or head trauma within 3 months; major surgery of head and spinal column within 2 months; visceral haemorrhage in the previous 4 weeks; major surgery or trauma in the previous 3 weeks; cardiopulmonary resuscitation which lasted $>10$ mins or endotracheal intubation; vascular punctures with haemostasis site unable to be compressed within 2 weeks; current therapy with warfarin, dabigatran, rivaroxaban or glycoprotein IIb/ 


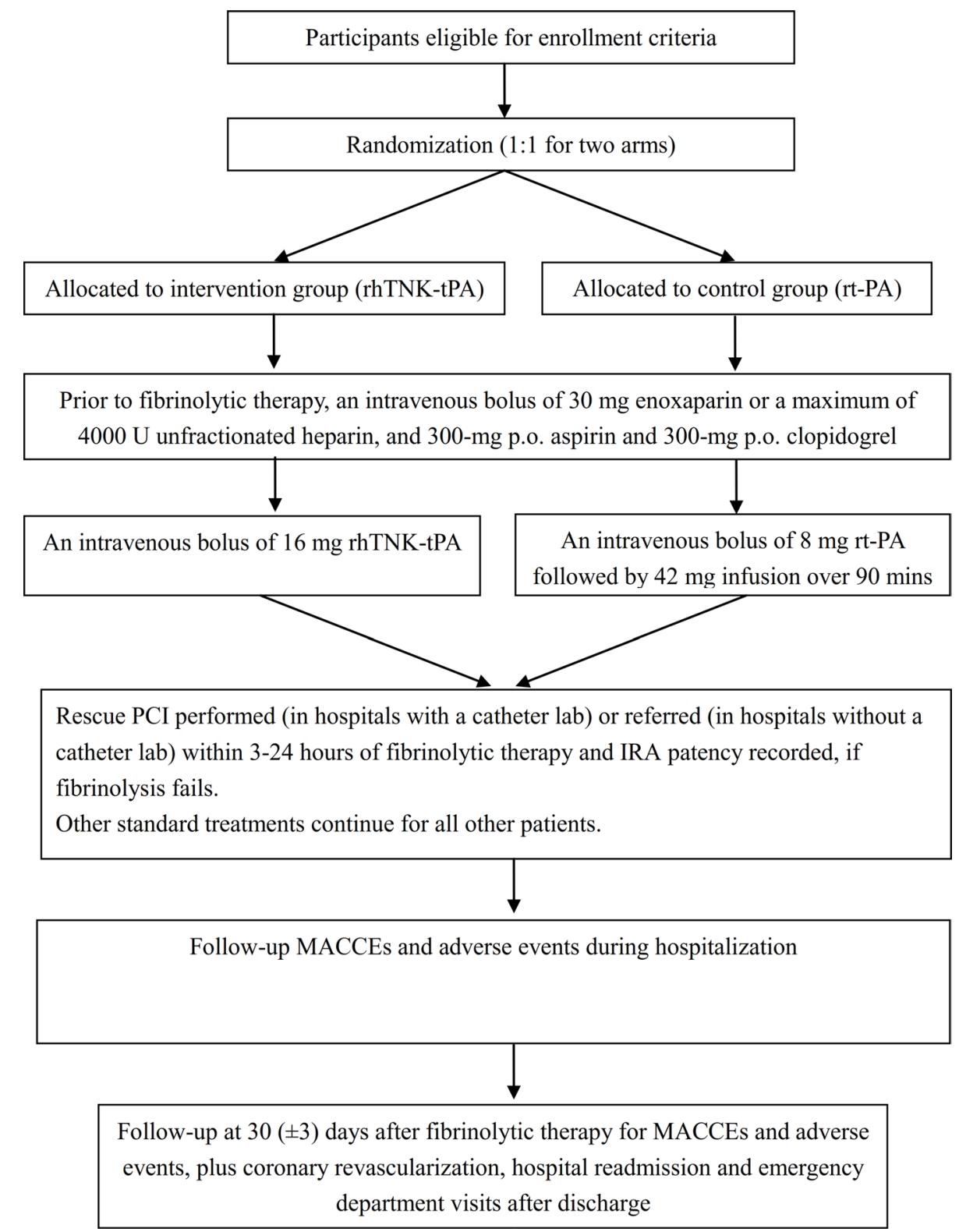

Figure 1 Recombinant human TNK tissue-type plasminogen activator (rhTNK-tPA) therapeutic efficacy non-inferiority trial flow chart. IRA, infarct-related artery; MACCEs, major adverse cardiovascular and cerebrovascular events; PCI, percutaneous coronary intervention; p.o., orally; rt-PA, recombinant human tissue-type plasminogen activator.

IIIa inhibitors; (3) having other major illnesses that would expose the subject to inordinate risk: indications of cardiac rupture; acute pericarditis, infective endocarditis, acute myocarditis, septic thrombophlebitis or severe infection accompanied by arteriovenous fistula; highly suspected thrombus in left heart chamber, such as mitral stenosis with atrial fibrillation; damage to the central nervous system, such as intracranial tumour, aneurysm, intracranial or spinal canal surgery; severe renal or hepatic dysfunction, or severe haematonosis; malignancy; diabetic haemorrhagic retinopathy or other haemorrhagic ophthalmopathy; history of PCI or coronary artery bypass grafting $(\mathrm{CABG})$; fibrinolytic therapy before admission; weight $<50 \mathrm{~kg}$; falling over or other trauma occurring after MI onset; (4) currently participating in another interventional trial; (5) allergic to rhTNK-tPA and/or rt-PA; (6) pregnancy or lactation; (7) inability or unwillingness to follow the protocol due to a mental disorder; and (8) any other conditions that, in the opinion of the investigators, make the potential participant unfit for involvement in the study.

Participants can withdraw from the research study at any time without any influence on future treatment. For every participant who withdraws from the study, the related information collected for early termination and the reasons for withdrawal will be recorded.

\section{Randomisation and blinding}

Central computerised randomisation (Medidata Balance) will be carried out by the Peking University Clinical Research Institute, which is independent of the research staff. Dynamic randomisation with varying block 
size will be stratified for research centre, age $(\leq 60$ years vs $>60$ years) and the duration from onset of symptoms to randomisation ( $\leq 3$ hours vs $3-6$ hours). The participant will be randomised to either the intervention group (rhTNK-tPA) or the control group (rt-PA) on a 1:1 ratio. The allocation sequence is computer-generated and the randomisation list is not known to the investigators.

Given the nature of the intervention, the participants and investigators cannot be blinded due to significant differences in administration for the intervention and control treatments. However, the primary outcome (major adverse cardiovascular events, MACCEs) and safety indicators will be evaluated by an independent Clinical Endpoint Committee (CEC), which is blinded to treatment assignment. In addition, coronary angiography results will be uniformly reviewed by the core laboratory, which is blinded to treatment allocation status, and TIMI flow grade will be determined accordingly.

\section{Study treatments}

All patients will receive concomitant antiplatelet and anticoagulant therapy before fibrinolytic therapy according to guideline-based clinical practice. In our study, enoxaparin is the primary drug for anticoagulant co-therapy; however, unfractionated heparin could be used instead of enoxaparin if enoxaparin is not available. Participants administered enoxaparin will receive an intravenous bolus of $30 \mathrm{mg}$ followed by (after 15 mins) a subcutaneous injection $(1.0 \mathrm{mg} / \mathrm{kg})$ which will be repeated every 12 hours for a maximum of 8 days. The first two subcutaneous doses should not exceed $100 \mathrm{mg}$. However, the subcutaneous dose will be repeated every 24 hours for participants whose creatinine clearance rate is $<30 \mathrm{~mL} /$ min. Participants administered unfractionated heparin will receive a loading dose of $4000 \mathrm{U}$ followed by fibrinolytic therapy and $12 \mathrm{U} / \mathrm{kg}$ per hour infusion (no more than $1000 \mathrm{U}$ /hour) to sustain activated partial thromboplastin time values within the range of 1.5 to 2.0 times the baseline control (50-70s) for 24-48hours. Subsequent heparin administration will be at the discretion of the investigator. Antiplatelet therapy consists of aspirin in a $300 \mathrm{mg}$ oral loading dose before fibrinolytic therapy followed by $100 \mathrm{mg}$ daily, and clopidogrel in a $300 \mathrm{mg}$ oral loading dose before fibrinolytic therapy followed by $75 \mathrm{mg}$ daily.

All participants assigned to the intervention group will receive an intravenous bolus of $16 \mathrm{mg}$ rhTNK-tPA. The participants assigned to the control group will receive an intravenous bolus of $8 \mathrm{mg}$ tPA followed by an infusion of $42 \mathrm{mg}$ in $90 \mathrm{mins}$, according to the dosing regimen in the TPA/Urokinase Comparisons in China (TUCC) trial. ${ }^{18}$

In addition to specified therapies in our study, other medications can also be administered at the discretion of the responsible physicians. The decision to proceed further with PCI after the fibrinolytic therapy is left to the investigator's judgement. Rescue PCI will be performed as soon as possible if fibrinolysis fails. Coronary angiography is suggested to be performed 3 to 24 hours after fibrinolytic therapy. The administered dose of unfractionated heparin could be adjusted to reach an activated clotting time of 250-300 s during the implementation of PCI in the catheterisation laboratory. Additional dosage of anticoagulant therapy is not necessary for rescue PCI if enoxaparin is used as the primary drug for concomitant therapy. However, if PCI is performed within 3 to 24 hours of receiving fibrinolytic therapy, an additional dosage of enoxaparin is also not necessary when the last subcutaneous dose is given within 8 hours, and an intravenous bolus of $0.3 \mathrm{mg} / \mathrm{kg}$ enoxaparin will be added when the last subcutaneous dose is given in 8 to 12 hours. In hospitals unable to implement coronary revascularisation, participants can be transferred to a collaborative tertiary hospital equipped with facilities to perform PCI or coronary angiography when appropriate.

All participants will be closely monitored within 24 hours of fibrinolytic therapy. During that period, a 12 lead electrocardiogram (18 lead ECG for posterior wall and right ventricular MI) examination will be repeated at 30,60 , 90 and 120 mins after fibrinolysis. When appropriate, an ECG examination could be done at the discretion of the responsible physicians. Clinical symptoms and signs should be evaluated, especially for duration and relief of chest pain. Creatine kinase-MB (CK-MB) and cardiac troponin (cTn) (if available) will be measured at 10, 12, 14, 16, 18 and 24 hours after symptom onset. TIMI flow grade will be evaluated and recorded if coronary angiography is done within 24 hours of fibrinolytic therapy. In addition, IRA patency will also be evaluated according to the non-invasive clinical indexes mentioned above within 24 hours of fibrinolytic therapy.

\section{Baseline assessment and follow-up}

The study will consist of three phases: baseline assessment, in-hospital follow-up, and follow-up after discharge. For each phase the main information collected is described below. Figure 2 provides an overview of the most important data.

\section{Baseline assessment}

After receiving written informed consent and checking the inclusion/exclusion criteria, the responsible cardiologist will need to collect the required data using a uniform electronic case report form (e-CRF). The data to be collected at baseline include demographic characteristics, physical examination, vital signs at hospital admission, history of present illness (onset time of chest pain, admission time and duration of chest pain), cardiac function with Killip class, past medical history (MI, hypertension, diabetes mellitus, hyperlipaemia, arrhythmia, peptic ulcer and stroke), past therapeutic history (PCI, CABG, medications for cardiovascular disease), smoking history, laboratory testing (blood routine, blood biochemistry, routine urine test and myocardial damage biomarker), 18 lead ECG, and adverse events. After randomisation, the participants will receive the corresponding planned treatment (rhTNK-tPA or rt-PA) and will be followed up 


\begin{tabular}{|c|c|c|c|c|}
\hline & $\begin{array}{l}\text { Baseline } \\
\text { screening }\end{array}$ & $\begin{array}{l}\text { Monitoring after } \\
\text { fibrinolysis }\end{array}$ & $\begin{array}{l}\text { In-hospital } \\
\text { follow-up }\end{array}$ & $\begin{array}{l}\text { 30-days } \\
\text { follow-up }\end{array}$ \\
\hline Informed consent & $\bullet$ & & & \\
\hline Checking enrollment criteria & $\bullet$ & & & \\
\hline Demographical characteristics & - & & & \\
\hline Medical history ${ }^{\dagger}$ & $\bullet$ & & & \\
\hline Physical examination & $\bullet$ & & & \\
\hline Vital signs & $\bullet$ & $\bullet$ & $\bullet$ & \\
\hline Clinical symptoms & $\bullet$ & $\bullet$ & $\bullet$ & \\
\hline Cardiac function ${ }^{\S}$ & $\bullet$ & & & \\
\hline Laboratory examination & - & $\bullet$ & $\bullet^{\zeta}$ & \\
\hline Electrocardiogram $^{\Psi}$ & $\bullet$ & $\bullet$ & $\bullet$ & \\
\hline CK-MB ${ }^{*}$ & $\bullet$ & $\bullet$ & $\bullet$ & \\
\hline Randomization & $\bullet$ & & & \\
\hline Drug administration & $\bullet$ & & & \\
\hline $\begin{array}{l}\text { IRA patency, heart failure and } \\
\text { cardiac shock }\end{array}$ & & - & • & \\
\hline $\begin{array}{l}\text { Coronary angiography and } \\
\text { ultrasonic cardiogram }\end{array}$ & & $\bullet$ & $\bullet$ & \\
\hline Coronary revascularization & & & $\bullet$ & $\bullet$ \\
\hline Concomitant medication & $\bullet$ & $\bullet$ & - & - \\
\hline MACCEs $^{\mathfrak{E}}$ & & • & • & - \\
\hline Medical direct expense & & & - & \\
\hline $\begin{array}{l}\text { Diagnosis and outcome of } \\
\text { hospital discharge }\end{array}$ & & & $\bullet$ & \\
\hline Adverse events & $\bullet$ & $\bullet$ & $\bullet$ & $\bullet$ \\
\hline Health care ${ }^{f_{3}}$ & & & & $\bullet$ \\
\hline
\end{tabular}

Figure 2 Baseline evaluation, fibrinolytic therapy, and follow-up schedule. ${ }^{\dagger}$ Including past medical history and past therapeutic

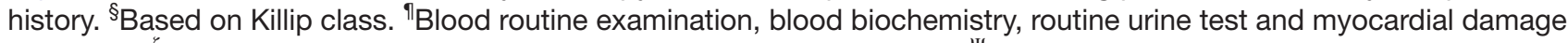
biomarker. 'Blood routine examination and myocardial damage biomarker. ${ }^{\Psi} 18$ lead electrocardiogram (ECG) before fibrinolytic therapy; 12 lead ECG (18 lead ECG for posterior wall and right ventricular MI) examination repeated at 30, 60, 90 and 120 mins after fibrinolysis; when appropriate, ECG examination could be done at the discretion of the responsible physicians. ${ }^{\ddagger}$ Detected at 10,12, 14, 16, 18 and 24 hours after symptom onset, and on the second and third day after hospital admission. If available, cardiac troponin (cTn) will be collected at the time points. ${ }^{\text {E}}$ Including mortality, non-fatal reinfarction, non-fatal stroke (both ischaemic and haemorrhagic stroke), percutaneous coronary intervention $(\mathrm{PCl})$ due to thrombolysis failure and $\mathrm{PCl}$ due to reocclusion. ${ }^{\complement}$ Hospital readmission and emergency department visiting due to cardiovascular disease. CK-MB, creatine kinaseMB; IRA, infarct-related artery; MACCEs, major adverse cardiovascular and cerebrovascular events.

based on the same schedule and procedures for both arms (figure 2). The assigned treatment, medication time of fibrinolytic therapy, antiplatelet and anticoagulant therapy, other concomitant medications and adverse events should be recorded in detail.

\section{In-hospital follow-up}

Before the patient's discharge, the responsible investigator will need to collect all data of relevance to identify and diagnose the study outcomes, using a uniform e-CRF. Please refer to the following section of primary and secondary outcomes for further details.

\section{Thirty days follow-up}

All randomised participants are planned to be followed-up at $30( \pm 3)$ days after fibrinolysis, with both face-to-face interview and telephone follow-up acceptable. Please refer to the following section of primary and secondary outcomes for further details.

\section{Study endpoints}

The primary study endpoint is the occurrence of MACCEs within 30 days of fibrinolytic therapy, including all-cause mortality, non-fatal reinfarction, non-fatal stroke (both ischaemic and haemorrhagic), PCI due to thrombolysis failure, and PCI due to reocclusion. MACCEs will be adjudicated by an independent CEC which is blinded to treatment allocation.

Secondary endpoints include the following: (a) TIMI flow grade 3 in the IRA within 24 hours of fibrinolytic therapy according to coronary angiography (to be restricted to participants with available coronary angiography within 24 hours of fibrinolytic therapy). (b) IRA patency within 24 hours of fibrinolytic therapy diagnosed by non-invasive clinical indexes; ${ }^{19}{ }^{20}$ IRA patency can be determined if any two of the following four items $(3+4$ excluded) can be achieved: (1) reduction of elevated ST-segment $\geq 50 \%$ by ECG is achieved within $60-90$ mins 
of receiving fibrinolytic therapy; (2) the time to peak cTn concentration is advanced to $\leq 12$ hours of symptom onset and the time to CK-MB concentration is advanced to $\leq 14$ hours of symptom onset; (3) significant relief of chest pain within 2 hours of fibrinolytic therapy; (4) presence of reperfusion arrhythmia within 2 to 3 hours of fibrinolytic therapy, including accelerated idioventricular rhythm, sudden improvement or disappearance of atrioventricular block or bundle branch block, and transient sinus bradycardia or sino-auricular block with or without hypotension among patients with inferior wall MI. (c) The occurrence of MACCEs during hospitalisation. (d) All-cause mortality during hospitalisation and within 30 days of fibrinolytic therapy. (e) Cardiovascular mortality during hospitalisation and within 30 days of fibrinolytic therapy. (f) The occurrence of reinfarction during hospitalisation. (g) The occurrence of new onset or worsened heart failure during hospitalisation. (h) The occurrence of cardiac shock during hospitalisation. (i) The occurrence of coronary revascularisation within 30 days of fibrinolytic therapy.

Safety endpoints include the following: (a) The occurrence of intracranial haemorrhage during hospitalisation. (b) The occurrence of gastrointestinal tract major bleeding during hospitalisation. (c) Bleeding events by severity during hospitalisation. (d) The frequency and severity of adverse events. Bleeding events will be assessed according to TIMI bleeding criteria and adjudicated by the CEC. Major bleeding is defined as any intracranial bleeding, or clinically significant haemorrhage that is relevant with a $\geq 5 \mathrm{~g} / \mathrm{dL}$ drop in haemoglobin $(\mathrm{Hb})$ or a $\geq 15$ point decrease in haematocrit. Minor bleeding is defined as any clinically significant haemorrhage (including imaging) that is relevant with $3-5 \mathrm{~g} / \mathrm{dL}$ drop in $\mathrm{Hb}$ and 9-14 point decrease in haematocrit. ${ }^{21}$ Other bleeding events which do not meet the above two bleeding criteria are classified as minimal bleeding.

In addition, a pharmacoeconomic evaluation will be carried out in our study; this will include the direct medical expenses for the first hospitalisation which can be obtained directly from hospital records; the number of hospital readmissions; and the frequency of visits to the emergency department due to cardiovascular disease within 30 days of fibrinolytic therapy.

\section{Safety data monitoring}

The CEC is composed of four cardiovascular specialists and one neurological expert who are recommended and appointed by the Peking University Clinical Research Institute. The CEC is blinded to treatment assignment and independently evaluates the primary outcome and safety indicators. In addition, two secretaries help to collect supporting information about endpoint assessment, organise the communication of the evaluation committee, and summarise the final results.

Safety data, especially major bleeding events in both groups, will be reported to the Data and Safety Monitoring Committee (DSMB). As this trial is a non-inferiority study, an interim analysis is not planned and statistical criteria for premature termination will not be considered. However, throughout the trial period, the DSMB may review the safety data and will independently evaluate the safety data collected and any unexpected events according to the rules and guidelines of the DSMB. The safety data analyses should be done after 10\%, 25\% and $50 \%$ of patients have completed the study procedure, and the DSMB may make recommendations to continue, modify or terminate the study at any time based on safety concerns.

\section{Sample size estimation}

Data from our unpublished CPACS-3 study in China showed that the incidence of MACCEs was $17.2 \%$ among STEMI patients within 30 days of fibrinolytic therapy. We therefore assumed that the incidence of MACCEs would be $17 \%$ in the control group in this study, and we further set a $3 \%$ absolute rate increase as the non-inferiority margin, corresponding to a non-inferiority relative risk margin of 1.176. With these assumptions, 2923 participants in each arm of the study are required to provide $80 \%$ power with a $5 \%$ significance level using a two-tailed test. Assuming a 5\% loss to follow-up rate, enrolment of 6200 patients (or 3100 participants per arm) is projected to yield the necessary number of events.

\section{Statistical consideration}

Analyses will be made using SAS statistical software (version 9.4, SAS Institute, Cary, NC, USA). Baseline characteristics will be reported as frequencies (percentages) or mean \pm SD. The Student t-test or Wilcoxon rank-sum test will be used to compare the differences in the quantitative baseline characteristics between the intervention and control groups. Comparisons on qualitative variables will be done using the $\chi^{2}$ test or Fisher's exact test, as appropriate. A value of $\mathrm{p}<0.05$ at the two-tailed test is set as the statistically significant level.

Both intention-to-treat (ITT) and per-protocol analyses will be done for the primary analyses as is recommended for non-inferiority studies, but principally with reference to per-protocol analysis. Based on the ITT principle, a full analysis set (FAS) consisting of all the randomised patients will be used. Survival curves of MACCEs within 30 days of fibrinolytic therapy will be estimated by the KaplanMeier curve and compared by log-rank test. The occurrence of MACCEs within 30 days of fibrinolytic therapy will be compared with the $\chi^{2}$ test or Fisher's exact test as required, and a multivariate logistic regression model used to determine the independent difference between the two groups after adjusting for the impact of baseline characteristics on treatment effect.

The $\chi^{2}$ test or Fisher's exact test will be used to compare the difference of secondary endpoints between the two groups. If inequality of baseline characteristics is detected, the confounding factors will be defined and a multivariate logistic regression model will be used to adjust for covariate effects. Adverse events in the two 
arms, including bleeding events, will also be compared using the $\chi^{2}$ test or Fisher's exact test as required.

\section{Study status}

The first participant was enrolled on 11 July 2016. As of March 2017, recruitment is ongoing at 31 centres in China with a total of 32 patients randomised. Treatment and follow-up of all participants are planned to continue until December 2020.

\section{DISCUSSION}

As far as we know, this is currently the first randomised controlled trial to evaluate the efficacy and safety of rhTNK-tPA in comparison with rt-PA as fibrinolytic therapy in STEMI patients in China. PCI use is considerably hampered by several non-system and system barriers in China. Fibrinolytic therapy remains an important option for those STEMI patients with poor access to healthcare. The efficacy and safety of tenecteplase has been determined in previous studies in TIMI $10 \mathrm{~A},{ }^{22}$ TIMI $10 \mathrm{~B}^{23}$ and ASSENT-1 study. ${ }^{14}$ Therefore, the efficacy and safety of rhTNK-tPA (Recomlyse) can be assumed to be ideal as it has the same amino acid sequence as tenecteplase. Once the efficacy and safety of rhTNK-tPA is confirmed in this study, it should provide additional benefit for STEMI patients in China, especially for those patients with poor access to healthcare.

rhTNK-tPA is a genetically engineered variant of rt-PA, and has been developed to avoid some of the limitations of rt-PA. Similar to rt-PA, rhTNK-tPA has amino acid substitutions at three sites: adding a glycosylation site to position 103, cutting out a glycosylation site at position 117 , and replacing four amino acids with four alanines at the third position. ${ }^{23}$ There are several potential advantages for rhTNK-tPA generated by these substitutions, including a slower plasma clearance, better fibrin specificity, and higher resistance to plasminogen-activator inhibitor type 1 compared with rt-PA. ${ }^{152} 23$ Moreover, its bolus administration should facilitate rapid and complete administration, reduce the rate of medication errors, and make the strategy of pre-hospital thrombolysis more promising and feasible. ${ }^{24}$

The ASSENT-2 trial, in which 16949 participants from more than 1000 hospitals in 29 countries were randomised, showed that a single-bolus injection of tenecteplase and a rapid infusion of alteplase had an equivalent effect on 30-day mortality $(6.18 \%$ vs $6.15 \%) .{ }^{14}$ However, compared with participants in the alteplase group, those in the tenecteplase group had fewer major bleeding events $(4.66 \%$ vs $5.94 \%, \mathrm{p}<0.001)$ other than intracranial haemorrhage $(0.93 \%$ vs $0.94 \%) .{ }^{14}$ The lower risk of non-cerebral bleedings persisted in subgroups with different levels of risk. The almost identical rates of 30-day mortality and the lower risk of non-cerebral major bleedings showed that tenecteplase offered a safety benefit over alteplase in the treatment of STEMI patients.
Pharmacoeconomic evaluation will be carried out in our study, including direct medical expense for the first hospitalisation, the number of hospital readmissions, and the frequency of visits to the emergency department. Economic analysis will be implemented immediately after the time that clinical data are available. From a public health perspective, the study will serve as an important step towards understanding the cost-effectiveness of fibrinolytic therapy. The results of the economic analysis can provide important reference data to make budgetary and healthcare resource allocation decisions, particularly considering the high prevalence and seriousness of STEMI in China. Therefore, in addition to efficacy and safety data, the economic analysis for fibrinolytic therapy among STEMI patients should have great significance for clinicians and healthcare policymakers.

Treatment allocation cannot be blinded to either participants or investigators due to obvious differences in the administration methods between the intervention and control groups, which may lead to biased results. However, to prevent potential observer bias in assessment, the primary outcome (MACCEs) will be evaluated by an independent CEC which is blinded to treatment assignment. Moreover, data analysis will be carried out by a statistician at the Peking University Clinical Research Institute who is independent of the trial.

If the efficacy and safety of rhTNK-tPA is confirmed in this study, its application in China should help to improve the treatment of STEMI patients based on its potential advantages, including ease of bolus administration, longer plasma half-life, better fibrin specificity, and increased resistance to plasminogen-activator inhibitor.

\section{Author affiliations}

${ }^{1}$ Peking University Clinical Research Institute, Beijing, China

${ }^{2}$ Shenzhen-Peking University-The Hongkong University of Science \& Technology Medical Center, Shenzhen, China

${ }^{3}$ Department of Cardiology, Beijing Jishuitan Hospital, The Fourth Clinical Medical College of Peking University, Beijing, China

${ }^{4}$ Department of Cardiology, Fuwai Hospital, National Center for Cardiovascular Diseases, Chinese Academy of Medical Science and Peking Union Medical College, Beijing, China

${ }^{5}$ Guangzhou Recomgen Biotech Co., Ltd, Guangzhou, China

Acknowledgements The authors would like to acknowledge all clinical investigators for their great effort in the study implementation and all study participants.

Contributors HBW, PJ, XSZ, HYX, QY, YFW and SBQ developed the protocol and grant proposal for this project and wrote the manuscript. XYY, CY, and RLG contributed to the protocol and grant proposal. XYY, HYX and CY assisted with writing and editing of the manuscript. The manuscript was amended based on comments from all authors. All authors read and approved the final manuscript.

Funding This study is supported by Guangzhou Recomgen Biotech Co., Ltd.

Competing interests Guangzhou Recomgen Biotech Co., Ltd sponsored the clinical study. HBW, PJ, XYY, CY and YFW were responsible for study design and data analysis at the Peking University Clinical Research Institute, without direct payment from Guangzhou Recomgen Biotech Co., Ltd. XSZ, HYX, RLG and SBQ were clinical investigators in this clinical trial, without direct payment from Guangzhou Recomgen Biotech Co., Ltd for their roles in conducting the study. QY is the employee of Guangzhou Recomgen Biotech Co., Ltd. Principal investigator has full access to the final trial data set, but the sponsor does not have access to the data.

Patient consent Obtained. 
Ethics approval Institutional Review Boards of Fuwai Hospital, Chinese Academy of Medical Sciences in Beijing, China.

Provenance and peer review Not commissioned; externally peer reviewed.

Open Access This is an Open Access article distributed in accordance with the Creative Commons Attribution Non Commercial (CC BY-NC 4.0) license, which permits others to distribute, remix, adapt, build upon this work non-commercially, and license their derivative works on different terms, provided the original work is properly cited and the use is non-commercial. See: http://creativecommons.org/ licenses/by-nc/4.0/

(C) Article author(s) (or their employer(s) unless otherwise stated in the text of the article) 2017. All rights reserved. No commercial use is permitted unless otherwise expressly granted.

\section{REFERENCES}

1. GBD 2013 Mortality and Causes of Death Collaborators. Global, regional, and national age-sex specific all-cause and causespecific mortality for 240 causes of death, 1990-2013: a systematic analysis for the Global Burden of Disease Study 2013. Lancet 2015;385:117-71.

2. Zhou M, Wang H, Zhu J, et al. Cause-specific mortality for 240 causes in China during 1990-2013: a systematic subnational analysis for the Global Burden of Disease Study 2013. Lancet 2016;387:251-72.

3. O'Gara PT, Kushner FG, Ascheim DD, et al. 2013 ACCF/AHA guideline for the management of ST-elevation myocardial infarction: a report of the American College of Cardiology Foundation/American Heart Association Task Force on Practice Guidelines. J Am Coll Cardiol 20132013;61:e78-140.

4. Li J, Li X, Wang Q, et al. ST-segment elevation myocardial infarction in China from 2001 to 2011 (the China PEACE-Retrospective Acute Myocardial Infarction Study): a retrospective analysis of hospital data. Lancet 2015;385:441-51.

5. Viikilä J, Lilleberg J, Tierala I, et al. Outcome up to one year following different reperfusion strategies in acute ST-segment elevation myocardial infarction: the Helsinki-Uusimaa Hospital District registry of ST-Elevation Acute Myocardial Infarction (HUS-STEMI). Eur Heart J Acute Cardiovasc Care 2013;2:371-8.

6. Taylor J. 2012 ESC Guidelines on acute myocardial infarction (STEMI). Eur Heart J 2012;33:2501-2.

7. Keeley EC, Boura JA, Grines CL. Primary angioplasty versus intravenous thrombolytic therapy for acute myocardial infarction: a quantitative review of 23 randomised trials. Lancet 2003;361:13-20.

8. Boersma E. Primary Coronary Angioplasty vs. Thrombolysis Group. Does time matter? A pooled analysis of randomized clinical trials comparing primary percutaneous coronary intervention and inhospital fibrinolysis in acute myocardial infarction patients. Eur Heart J 2006;27:779-88.

9. Rathore SS, Curtis JP, Chen J, et al. Association of door-to-balloon time and mortality in patients admitted to hospital with ST elevation myocardial infarction: national cohort study. BMJ 2009;338:b1807.

10. Kushner FG, Hand M, Smith SC, et al. 2009 focused updates: ACC/ AHA guidelines for the management of patients with ST-elevation myocardial infarction (updating the 2004 guideline and 2007 focused update) and ACC/AHA/SCAI guidelines on percutaneous coronary intervention (updating the 2005 guideline and 2007 focused update) a report of the American College of Cardiology Foundation/American Heart Association Task Force on Practice Guidelines. J Am Coll Cardiol 2009;54:2205-41.

11. Bradley EH, Nallamothu BK, Herrin J, et al. National efforts to improve door-to-balloon time results from the Door-to-Balloon Alliance. J Am Coll Cardiol 2009;54:2423-9.

12. Xavier D, Pais P, Devereaux PJ, et al. Treatment and outcomes of acute coronary syndromes in India (CREATE): a prospective analysis of registry data. Lancet 2008;371:1435-42.

13. Armstrong PW, Gershlick AH, Goldstein P, et al. Fibrinolysis or primary PCl in ST-segment elevation myocardial infarction. $N$ Engl $J$ Med 2013;368:1379-87.

14. Van De Werf F, Adgey J, Ardissino D, et al. Single-bolus tenecteplase compared with front-loaded alteplase in acute myocardial infarction: the ASSENT-2 double-blind randomised trial. Lancet 1999;354:716-22.

15. Keyt BA, Paoni NF, Refino CJ, et al. A faster-acting and more potent form of tissue plasminogen activator. Proc Natl Acad Sci U S A 1994;91:3670-4.

16. Assessment of the Safety and Efficacy of a New Thrombolytic Regimen (ASSENT)-3 Investigators. Efficacy and safety of tenecteplase in combination with enoxaparin, abciximab, or unfractionated heparin: the ASSENT-3 randomised trial in acute myocardial infarction. Lancet 2001;358:605-13.

17. Zhai M, Chen JL, Qiao SB, et al. Efficacy and safety of recombinant human TNK tissue-type plasminogen activator in patients with acute myocardial infarction. Chinese Journal of New Drugs 2016;25:82-6.

18. Ross AM, Gao R, Coyne KS, et al. A randomized trial confirming the efficacy of reduced dose recombinant tissue plasminogen activator in a Chinese myocardial infarction population and demonstrating superiority to usual dose urokinase: the TUCC trial. Am Heart $J$ 2001;142:244-7.

19. Davies $\mathrm{CH}$, Ormerod OJ. Failed coronary thrombolysis. Lancet 1998;351:1191-6.

20. Board CJoCE. Reference scheme for treatment of fibrinolytic therapy for acute myocardial infarction. Chin Med J 1996;24:328-9.

21. Gibson CM, Mehran R, Bode C, et al. An open-label, randomized, controlled, multicenter study exploring two treatment strategies of rivaroxaban and a dose-adjusted oral vitamin $\mathrm{K}$ antagonist treatment strategy in subjects with atrial fibrillation who undergo percutaneous coronary intervention (PIONEER AF-PCI). Am Heart J 2015;169:472-8.

22. Cannon $\mathrm{CP}, \mathrm{McCabe} \mathrm{CH}$, Gibson $\mathrm{CM}$, et al. TNK-tissue plasminogen activator in acute myocardial infarction. Results of the Thrombolysis in Myocardial Infarction (TIMI) 10A dose-ranging trial. Circulation 1997;95:351-6.

23. Cannon CP, Gibson CM, McCabe CH, et al. TNK-tissue plasminogen activator compared with front-loaded alteplase in acute myocardial infarction: results of the TIMI 10B trial. Thrombolysis in Myocardial Infarction (TIMI) 10B Investigators. Circulation 1998;98:2805-14.

24. Weaver WD, Cerqueira M, Hallstrom AP, et al. Prehospital-initiated vs hospital-initiated thrombolytic therapy. The Myocardial Infarction Triage and Intervention Trial. JAMA 1993;270:1211-6. 